\title{
Management of axitinib (AG-013736)-induced fatigue and thyroid dysfunction, and predictive biomarkers of axitinib exposure: results from phase I studies in Japanese patients
}

\author{
Yutaka Fujiwara $\cdot$ Naomi Kiyota $\cdot$ Naoko Chayahara $\cdot$ \\ Akiyuki Suzuki • Yoshiko Umeyama • Toru Mukohara • \\ Hironobu Minami
}

Received: 8 December 2010 /Accepted: 24 January 2011 /Published online: 8 February 2011

(C) The Author(s) 2011. This article is published with open access at Springerlink.com

Summary Background Axitinib is an oral, potent and
selective inhibitor of vascular endothelial growth factor
receptors (VEGFRs) 1,2 and 3. We report on data obtained
from 18 Japanese patients with advanced solid tumors in
two phase I trials that evaluated the safety, pharmacokinet-
ics and antitumor activity of axitinib and also examined
potential biomarkers. Methods Six patients received a
single 5-mg dose of axitinib followed by $5 \mathrm{mg}$ twice daily
(BID), and an additional six patients received axitinib $5 \mathrm{mg}$
BID only. Another six patients received axitinib at 5 -mg,
7-mg and 10-mg single doses followed by $5 \mathrm{mg}$ BID.
Results Plasma pharmacokinetics following single doses of

Prior presentation The work presented in this manuscript is original and has not been published elsewhere. Some of the data have been presented previously in: Y. Fujiwara, N. Kiyota, N. Chayahara, et al. Effect of axitinib (AG-013736) on thyroid function and biomarkers: Results from phase I studies in Japanese patients. Poster presentation at 2009 AACR-EORTC-NCI Molecular Targets and Cancer Therapeutics Conference, Boston, Massachusetts, USA, November 15-19, 2009 (abstract B15).

Y. Fujiwara $(\bowtie) \cdot$ N. Kiyota $\cdot$ N. Chayahara $\cdot$ H. Minami Medical Oncology/Hematology, Department of Medicine,

Kobe University Hospital,

7-5-2, Kusunoki-cho,

Chuo-ku, Kobe 650-0017, Japan

e-mail: yufujiwa@med.kobe-u.ac.jp

A. Suzuki · Y. Umeyama

Pfizer Japan Inc,

3-22-7, Yoyogi,

Shibuya-ku, Tokyo, Japan

T. Mukohara $\cdot$ H. Minami

Kobe University Hospital,

Cancer Center, 7-5-2, Kusunoki-cho,

Chuo-ku, Kobe, Japan axitinib was generally linear. Common treatment-related adverse events were fatigue (83\%), anorexia (72\%), diarrhea (67\%), hand-foot syndrome (67\%) and hypertension $(61 \%)$. Sixteen patients $(89 \%)$ experienced thyroidstimulating hormone (TSH) elevation. Grade $3 / 4$ toxicities included hypertension (33\%) and fatigue (28\%). No grade $3 / 4$ fatigue occurred in patients who started thyroid hormone replacement therapy when TSH was elevated. Thyroglobulin elevation was observed in all patients who continued treatment with axitinib for $\geq 3$ months. Abnormal TSH correlated with exposure to axitinib $(r=0.72)$. Decrease in soluble (s) VEGFR-2 levels significantly correlated with exposure to axitinib $(r=-0.94)$. Axitinib showed antitumor activity across multiple tumor types. Conclusions Axitinib-related thyroid dysfunction could be due to a direct effect on the thyroid gland. Grade 3/4 fatigue and hypothyroidism appear to be controllable with use of thyroid hormone replacement therapy. sVEGFR-2 and TSH may act as biomarkers of axitinib plasma exposure.

Keywords Axitinib - Fatigue - Thyroid dysfunction .

Biomarker Pharmacokinetics - Vascular endothelial growth factor receptor

\section{Introduction}

Axitinib (AG-013736) is an oral, potent and selective inhibitor of vascular endothelial growth factor (VEGF) receptors (VEGFRs) 1, 2 and 3. Inhibition of the associated receptor tyrosine kinases is seen at picomolar concentrations [1]. Inhibition of the VEGF/VEGFR pathway by axitinib reduces VEGF-stimulated receptor autophosphorylation, blocking the 
growth and survival of endothelial cells, reducing tumor vascularization and inducing cell death [2]. Antitumor effects have been documented in a range of preclinical models, using single-agent axitinib or in combination with chemotherapy [3, 4]. Clinical activity with axitinib monotherapy, at a recommended starting dose of $5 \mathrm{mg}$ twice daily (BID), has been observed in phase II studies in patients with metastatic renal cell carcinoma (mRCC) refractory to treatment with cytokines [5] or sorafenib [6]. Two multicenter phase III studies of axitinib in advanced RCC are ongoing. Antitumor activity also has been noted in trials of axitinib in patients with advanced non-small cell lung cancer (NSCLC) [7], thyroid cancer [8], melanoma [9] and breast cancer [10].

A phase I study (Study 1) was conducted in 12 Japanese patients with solid tumors to determine the recommended starting dose for this patient population. This study also investigated the safety, pharmacokinetics following axitinib 5-mg single dosing and continuous dosing, the antitumor activity and potential biomarkers associated with singleagent axitinib [11]. Subsequently, a second phase I study (Study 2) was conducted in an additional six Japanese patients with solid tumors to evaluate pharmacokinetics following axitinib 5-mg, 7-mg, 10-mg single dosing and continuous dosing. In Study 1, fatigue and abnormal thyroidstimulating hormone (TSH) levels were frequently observed in 12 patients. In Study 2, therefore, free T3, free T4 and thyroglobulin were prospectively measured to further evaluate the effects of axitinib on the thyroid. Study 2 also explored biomarkers and antitumor activity. In this report, we newly reported the axitinib pharmacokinetic profile following $5-\mathrm{mg}$, 7-mg and 10-mg single dosing, thyroid function tests for free $\mathrm{T} 3$, free $\mathrm{T} 4$ and thyroglobulin, and management of fatigue with thyroid hormone replacement therapy. In addition, we updated safety data, antitumor activity, and the relationship between TSH level or soluble (s) VEGFR-2 versus axitinib exposure in 18 patients compared with the 12 patients evaluated in the initial phase I study (Study 1) [11].

The objectives of this analysis were to (1) evaluate the safety of axitinib in Japanese patients, including effects on fatigue and thyroid function; (2) evaluate plasma pharmacokinetics following axitinib 5-mg, 7-mg and 10-mg single and continuous dosing; (3) investigate plasma levels of VEGF, sVEGFR-2, sVEGFR-3 and soluble stem cell factor receptor (sKIT) and their potential as biomarkers; and (4) assess the preliminary antitumor activity of axitinib in this patient population.

\section{Methods}

Patient population

Patients were eligible for these studies if they had histologically or cytologically diagnosed advanced malignant solid tumors and were either ineligible for, or refractory to, standard therapies; aged 20-75 years in Study 1 and $\geq 20$ years in Study 2 ; expected survival $\geq 3$ months; adequate bone marrow, renal and hepatic function; Eastern Cooperative Oncology Group performance status (ECOG PS) 0,1 or 2 ; and no proteinuria (dipstick value $<1+$ or $<500 \mathrm{mg}$ per 24 -h urine collection if $\geq 1+$ in Study 1 and $<1+$ or $<2$ g per 24 -h urine collection if $\geq 1+$ in Study 2).

Patients with uncontrolled hypertension at screening (defined as blood pressure [BP] $>130 / 80 \mathrm{~mm} \mathrm{Hg}$ in Study 1 and $>140 / 90 \mathrm{~mm} \mathrm{Hg}$ in Study 2) were excluded, although use of antihypertensive agents was permitted. No anticancer therapy in the previous 4 weeks was allowed and no hormonal therapy, radiotherapy, oral fluorinated pyrimidine drug or a molecularly targeted agent was permitted for $\geq 2$ weeks prior to initiation of axitinib.

Other key exclusion criteria included the presence of central lung lesions involving major blood vessels; active gastrointestinal (GI) bleeding or GI disorders that could affect axitinib ingestion or absorption; active seizure disorder or brain metastases with symptoms or requiring treatment; cardiovascular disease in the previous 12 months, including myocardial infarction, severe or unstable angina, coronary artery/peripheral artery bypass graft or symptomatic congestive heart failure; cerebrovascular disorders, such as transient cerebral ischemic attack, deep vein thrombosis or pulmonary embolus, in the previous 12 months; and grade $\geq 3$ hemorrhage within 4 weeks or hemoptysis $(>2.5 \mathrm{~mL}$ bright-red blood per day) within the week prior to enrollment. Patients were also excluded if they were receiving or likely to receive drugs or food that could act as potent cytochrome P450 (CYP) $3 \mathrm{~A} 4$ inhibitors or CYP3A4 or CYP1A2 inducers, as were patients requiring anticoagulant therapy.

Study protocols were approved by the Institutional Review Board of the National Cancer Center, Tokyo, Japan (Study 1) and Kobe University Hospital, Kobe, Japan (Study 2). All patients provided written informed consent. The studies were conducted in accordance with the Declaration of Helsinki and the International Conference on Harmonization guidelines on Good Clinical Practice, as well as all applicable local regulatory requirements and laws.

The trials reported here are registered on the clinical trials site of the US National Cancer Institute website (Study 1, http:// www.clinicaltrials.gov/ct/show/NCT00447005; Study 2, http://www.clinicaltrials.gov/ct/show/NCT00726752).

\section{Drug administration}

In Study $1(N=12)$, the first six patients received a single 5 -mg dose of axitinib. If well tolerated over the initial $48 \mathrm{~h}$, they received continuous axitinib $5 \mathrm{mg}$ BID for the first 28-day cycle (Fig. 1). Axitinib single 5-mg dosing was administered to the first 6 patients only in order to evaluate 
Fig. 1 Study schemas. BID, twice daily. ${ }^{a} 5 \mathrm{mg}$ BID could be titrated up to $7 \mathrm{mg}$ BID, then $10 \mathrm{mg}$ BID or interrupted/ reduced to $3 \mathrm{mg}$ BID, then $2 \mathrm{mg}$ BID. ${ }^{\mathrm{b}}$ All patients in Study 2 received axitinib at 5-mg, $7-\mathrm{mg}$ and $10-\mathrm{mg}$ single doses on days $-5,-3$ and -1

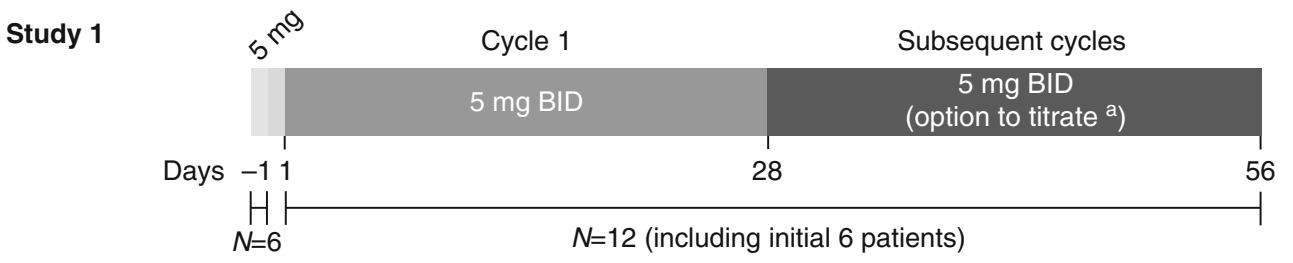

Study 2

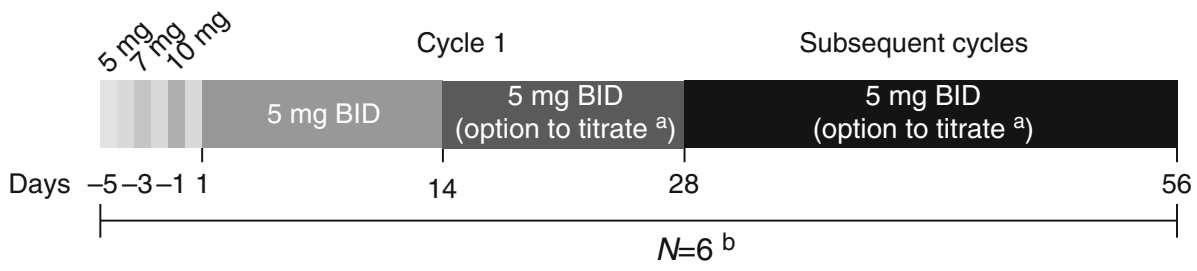

axitinib terminal-phase plasma half-life. The observation period (48 h) was set to evaluate the pharmacokinetics of axitinib up to $32 \mathrm{~h}$ after axitinib single 5-mg dosing. This observation period was also used in the first-in-human (FIH) study previously conducted in Caucasian patients. The same observation period was used to maintain consistency between both phase I studies conducted in Western and Japan. If axitinib was well tolerated with no treatment-related grade $>2$ adverse events occurring over a consecutive 2 -week period in subsequent cycles, the dose of axitinib could be increased to $7 \mathrm{mg}$ BID and then to a maximum $10 \mathrm{mg}$ BID continuous dosing unless systolic BP (sBP) was $>150 \mathrm{~mm} \mathrm{Hg}$, diastolic $\mathrm{BP}(\mathrm{dBP})>90 \mathrm{~mm} \mathrm{Hg}$ and the patient was receiving antihypertension medication. The axitinib dose could be reduced to $3 \mathrm{mg}$ BID and then to $2 \mathrm{mg}$ BID in the event of treatment-related toxicity or patient intolerance. An additional six patients in this study were started on continuous axitinib dosing at $5 \mathrm{mg}$ BID without receiving an initial single dose.

In Study $2(N=6)$, patients received a single dose of $5 \mathrm{mg}$ axitinib followed by a single dose of $7 \mathrm{mg}$ and then $10 \mathrm{mg}$ (Fig. 1). If these doses were safely administered, patients could receive continuous axitinib 5-mg BID dosing for the first 14 days. Subsequently, these patients also had the option of dose titration (increase or decrease) during the continuous dosing period.

In both studies, axitinib was administered orally in a fed state. A food effect study conducted in Western healthy volunteers demonstrated that lower plasma exposure of axitinib was obtained in the fed state versus overnight fasting state [12]. However, there was no difference in exposure of axitinib between the fed state and shorter fasting durations (i.e. $2 \mathrm{~h}$ before and $2 \mathrm{~h}$ after dosing, and $1 \mathrm{~h}$ before and $1 \mathrm{~h}$ after dosing, respectively). Since it was considered impractical for patients to be "overnight fasted" around each of the two daily doses of axitinib, and in order to avoid fluctuations in plasma exposures between morning and evening doses of the drug, axitinib administration in the fed state was recommended.
The axitinib dose was reduced or interrupted in both trials if drug-related toxicity occurred according to predefined dosemodification criteria. Axitinib treatment was interrupted or the dose was reduced if patients developed hemoptysis, hypertension ( $\mathrm{sBP}>150 \mathrm{~mm} \mathrm{Hg}$ or $\mathrm{dBP}>100 \mathrm{~mm} \mathrm{Hg}$ ) despite institution of maximal antihypertensive therapy or proteinuria ( $\geq 2 \mathrm{~g} / 24 \mathrm{~h}$ ). Patients continued axitinib therapy until disease progression, intolerable toxicity or withdrawal of consent.

Study assessments

\section{Safety}

Adverse events were assessed throughout the studies using Common Toxicity Criteria for Adverse Events, version 3.0. In Study 1, TSH measurements were performed every week in cycle 1, then every 2 weeks. In Study 2, antithyroperoxidase (anti-TPO) and antithyroglobulin antibody titers were determined at screening; TSH, free triiodothyronine (free T3), free thyroxine (free T4) and thyroglobulin were measured every week in cycle 1 and every 2 weeks thereafter. In addition, total cholesterol and creatine phosphokinase (CPK), which were potentially relevant to thyroid function, were also measured. Previous studies have found evidence of hypothyroidism in patients treated with other antiangiogenic agents, such as sunitinib, and related symptoms (including fatigue) improved with administration of thyroid hormone replacement therapy $[6,13]$. In Study 2, thyroid function-test values were prospectively monitored at every visit, and thyroid hormone replacement therapy was proactively given to patients, as appropriate according to TSH levels, to maintain a euthyroid state.

\section{Pharmacokinetics}

To assess axitinib pharmacokinetics, blood samples were collected from patients who received 5-mg, 7-mg and 10-mg single doses (pre-dose and 0.5, 1, 2, 4, 6, 8, 10, 24 and $32 \mathrm{~h}$ 
post dose) in Study 2. The blood samples were also collected during continuous dosing (pre-dose and 0.5, 1, 2, 4, 8 and $12 \mathrm{~h}$ post dose) at cycle 1 day 1 and cycle 1 day 15 in Study 1 and at cycle 1 day 15 in Study 2. Pharmacokinetic parameters evaluated for single doses included maximum plasma concentration $\left(\mathrm{C}_{\max }\right)$, area under the plasma concentrationtime curve $(\mathrm{AUC})$ from time zero to infinity $\left(\mathrm{AUC}_{\mathrm{inf}}\right)$, time to first occurrence of $\mathrm{C}_{\max }\left(\mathrm{T}_{\max }\right)$ and terminal-phase plasma half-life $\left(t_{1 / 2}\right)$. AUC over the dosing interval (AUC from time zero to $\left.12 \mathrm{~h}\left[\mathrm{AUC}_{12}\right]\right)$ and accumulation ratio $\left(\mathrm{R}_{\mathrm{ac}}\right)$ were also measured following continuous dosing.

Blood samples were centrifuged at $1,000 \times \mathrm{g}$ for $15 \mathrm{~min}$ and plasma was stored frozen at $-20^{\circ} \mathrm{C}$. Plasma samples were analyzed for axitinib concentration at Charles River Laboratories, Inc. (Worcester, MA, USA) using a validated high-performance liquid chromatography-tandem mass spectrometric method; the lower limit of quantification was $0.1 \mathrm{ng} / \mathrm{mL}$.

\section{Soluble biomarkers}

Plasma samples were collected to assess changes in soluble protein biomarkers that might be associated with axitinibinduced VEGF/VEGFR inhibition. Plasma concentrations of VEGF, sVEGFR-2, sVEGFR-3 and sKIT were measured in patient blood samples at baseline and during treatment on day 1 of each cycle up to 12 cycles and at the end of treatment. Each biomarker was measured using an enzymelinked immunosorbent assay (ELISA) kit at Alta Analysis (San Diego, CA, USA). Exploratory analyses were conducted to investigate the relationship between these endpoints and pharmacokinetics.

\section{Preliminary antitumor activity}

Antitumor activity was assessed at screening, every 8 weeks during continuous dosing and at the end of study, according to Response Evaluation Criteria in Solid Tumors (RECIST, version 1.0) [14].

\section{Results}

Patient demographics

Patient demographics and baseline characteristics for the two studies are shown in Table 1. Of the 18 patients enrolled in the two studies, the majority had an ECOG PS of 0 or 1 . The most common tumor type was colorectal cancer. Most patients were heavily pretreated; 14/18 patients $(78 \%)$ had received $\geq 3$ prior systemic treatment regimens. Six patients were receiving concurrent antihypertension medication at the start of axitinib therapy.
Table 1 Patient demographics and baseline characteristics (pooled data from Study 1 and Study 2)

\begin{tabular}{ll}
\hline & $N=18$ \\
\hline $\begin{array}{l}\text { Median age (range), y } \\
\text { Gender, } n(\%)\end{array}$ & $63(25-75)$ \\
Male & $10(56)$ \\
Female & $8(44)$ \\
ECOG PS, $n(\%)$ & \\
0 & $7(39)$ \\
1 & $10(56)$ \\
2 & $1(6)$ \\
Tumor type, $n(\%)$ & \\
Colorectal cancer & $6(33)$ \\
Renal cell cancer & $2(11)$ \\
Liposarcoma & $2(11)$ \\
Other ${ }^{\text {a }}$ & $8(44)$ \\
No. of prior systemic treatment regimens, $n(\%)$ & \\
1 & $3(17)$ \\
2 & $1(6)$ \\
3 & $5(28)$ \\
4 & $4(22)$ \\
$\geq 5$ & $5(28)$ \\
Yoncurrent antihypertensive agent, $n(\%)$ & $6(33)$ \\
No & $12(67)$ \\
\hline
\end{tabular}

\footnotetext{
${ }^{a}$ Ovarian cancer; non-small cell lung cancer; thymic cancer; synovial sarcoma; esophageal malignant melanoma; hypopharynx cancer; gastric cancer; pancreatic cancer
}

ECOG PS Eastern Cooperative Oncology Group performance status

Safety

Common treatment-related adverse events are shown in Table 2 and treatment-related laboratory abnormalities for the two studies are listed in Table 3. The most common adverse events (all grades) were fatigue, anorexia, diarrhea, hand-foot syndrome and hypertension. Grade 3/4 adverse events were infrequent and mainly limited to fatigue and hypertension. The most frequent treatment-related laboratory abnormality was increased TSH, but all cases were low-grade in severity. Proteinuria occurred in nine patients, with grade $3 / 4$ proteinuria seen in two patients.

\section{Pharmacokinetics}

Plasma pharmacokinetic parameters following single and continuous dosing of axitinib in Study $2(N=6)$ are shown in Table 4. The plasma pharmacokinetics following single dosing of $5 \mathrm{mg}, 7 \mathrm{mg}$ and $10 \mathrm{mg}$ axitinib seemed to be generally linear. The plasma axitinib concentration profiles 
Table 2 Common treatment-related adverse events (pooled data from Study 1 and Study 2; $N=18$ )

\begin{tabular}{lll}
\hline Adverse event & All grades, $n(\%)$ & Grade 3/4, $n(\%)$ \\
\hline Fatigue & $15(83)$ & $5(28)$ \\
Anorexia & $13(72)$ & 0 \\
Diarrhea & $12(67)$ & 0 \\
Hand-foot syndrome & $12(67)$ & $1(6)$ \\
Hypertension & $11(61)$ & $6(33)$ \\
Stomatitis & $10(56)$ & 0 \\
Hoarseness & $9(50)$ & 0 \\
Constipation & $7(39)$ & 0 \\
Rash & $6(33)$ & 0 \\
Epistaxis & $5(28)$ & 0 \\
Nausea & $4(22)$ & 0 \\
Headache & $4(22)$ & 0 \\
\hline
\end{tabular}

after single-dose administration are shown in Fig. 2. Following a single dose of axitinib, the plasma concentration reached a maximum $\left(\mathrm{C}_{\max }\right)$ at $\sim 4 \mathrm{~h}$ after administration and was eliminated with a half-life of 4.8-5.9 h. The mean (\% coefficient of variation) $\mathrm{R}_{\mathrm{ac}}$ after continuous dosing was $1.37(28 \%)$, consistent with the value predicted from the mean half-life of axitinib.

\section{Thyroid function}

In the two studies, 16 patients (89\%) experienced elevation of TSH above the upper limit of normal range. Changes in $\mathrm{TSH}$, free T3, free T4 and thyroglobulin levels over time for patients in Study 2 are shown in Fig. 3a-e. While the kinetics of response varied, all three patients who continued treatment with axitinib for $\geq 3$ months exhibited an increase in thyroglobulin levels between day 100 and day 200 following initiation of axitinib therapy, which subsequently returned to baseline (Fig. 3e). Normalization of thyroglobulin levels was associated with the elevation of TSH levels in these patients. One of six patients had positive antithyroglobulin antibody titers at baseline and another one patient tested positive for anti-TPO antibody in Study 2. Changes in thyroid function tests were observed in patients regardless of baseline antithyroglobulin/anti-TPO antibody status.

Thyroid hormone replacement therapy was proactively given to patients enrolled in Study 2, according to TSH levels measured at each visit, to maintain a euthyroid state. As shown in Table 5, while grade $3 / 4$ fatigue occurred in $42 \%$ of patients in Study 1, no cases of grade 3/4 fatigue were noted in Study 2. Proactive administration of thyroid hormone replacement therapy appeared to prevent the development of axitinib-related grade 3/4 fatigue.
In Study 2, a slight TSH decrease below the lower limit of normal range was observed in two patients between day 120 and day 170, and between day 320 and day 340 (Fig. 3b). This observation occurred during thyroid hormone replacement therapy and TSH decrease normalized with dose reduction or a temporary interruption of thyroid hormone replacement therapy.

In the three patients in Study 2 who received at least three cycles of axitinib treatment, changes in TSH levels mirrored the kinetics of change in total cholesterol levels. Meanwhile, no clear correlation was observed between TSH levels and CPK.

The relationship between changes in levels of TSH and axitinib plasma AUC was also investigated. When data for patients in both studies were analyzed, TSH change (baseline to cycle 2 day 1) was found to increase linearly with $\mathrm{AUC}_{12}$, with a correlation coefficient $r$ of $0.723(95 \%$ confidence interval $[\mathrm{CI}] 0.361$ to $1.000 ; P=0.0180$ ) (Fig. 4).

\section{Soluble plasma biomarkers}

Table 6 shows changes in plasma concentrations of VEGF, sVEGFR-2, sVEGFR-3 and sKIT from baseline to cycle 2 day 1 in both studies. Significant increases in median VEGF levels $(+242 \%)$ and decreases in median plasma sVEGFR-2 and sVEGFR-3 levels ( $-38 \%$ and $-53 \%$, respectively) were observed. An inverse relationship was seen between percent change in sVEGFR-2 levels (baseline to cycle 2 day 1) and axitinib $\mathrm{AUC}_{12}$ with a correlation coefficient $r$ of -0.940 (95\% CI -1.000 to $-0.841 ; P<0.0001)$ (Fig. 5).

Table 3 Common treatment-related laboratory abnormalities (pooled data from Study 1 and Study 2; N=18)

\begin{tabular}{lll}
\hline Adverse event & All grades, $n(\%)$ & Grade 3/4, $n(\%)$ \\
\hline $\begin{array}{l}\text { Non-hematologic } \\
\text { TSH increased }\end{array}$ & $13(72)$ & 0 \\
Proteinuria & $9(50)$ & $2(11)$ \\
Hematuria & $7(39)$ & 0 \\
Lipase increased & $6(33)$ & 0 \\
Blood glucose increased & $5(28)$ & 0 \\
ALP increased & $4(22)$ & $1(6)$ \\
AST increased & $4(22)$ & 0 \\
TSH decreased & $4(22)$ & 0 \\
Hematologic & & \\
Platelet count decreased & $6(33)$ & 0 \\
Neutrophil count decreased & $3(17)$ & $1(6)$ \\
Lymphocyte count & $1(6)$ & 0 \\
decreased & & \\
\hline
\end{tabular}

$A L P$ alkaline phosphatase; $A S T$ aspartate aminotransferase; TSH thyroid-stimulating hormone 
Table 4 Pharmacokinetic parameters following single and continuous dosing of axitinib (Study $2 ; N=6$ )

\begin{tabular}{|c|c|c|c|c|}
\hline \multicolumn{5}{|c|}{ Single dosing } \\
\hline \multirow[t]{2}{*}{ Dose } & Mean ${ }^{\mathrm{a}}(\% \mathrm{CV})$ & Mean $^{\mathrm{a}}(\% \mathrm{CV})$ & Mean $^{\mathrm{a}}(\% \mathrm{CV})$ & Median (min, max) \\
\hline & $\mathrm{C}_{\max }, \mathrm{ng} / \mathrm{mL}$ & $\mathrm{AUC}_{\text {Inf }}, \mathrm{ng} \cdot \mathrm{hr} / \mathrm{mL}$ & $t_{1 / 2}, h$ & $\mathrm{~T}_{\max }, \mathrm{h}$ \\
\hline $5 \mathrm{mg}$ & $17.0(70)$ & $142(86)$ & $4.8(59)$ & $4.10(3.95,6.02)$ \\
\hline $7 \mathrm{mg}$ & $23.3(88)$ & $181(80)$ & $5.1(51)$ & $4.00(0.983,9.88)$ \\
\hline $10 \mathrm{mg}$ & $34.9(115)$ & $288(91)$ & $5.9(59)$ & $4.02(2.05,6.00)$ \\
\hline \multicolumn{5}{|c|}{ Continuous dosing (cycle 1 day 15 ) } \\
\hline \multirow[t]{2}{*}{ Dose } & Mean $^{\mathrm{a}}(\% \mathrm{CV})$ & Mean $^{\mathrm{a}}(\% \mathrm{CV})$ & Median (min, max) & Mean ${ }^{\mathrm{a}}(\% \mathrm{CV})$ \\
\hline & $\mathrm{C}_{\max }, \mathrm{ng} / \mathrm{mL}$ & $\mathrm{AUC}_{12}, \mathrm{ng} \cdot \mathrm{hr} / \mathrm{mL}$ & $\mathrm{T}_{\max }, \mathrm{h}$ & $\mathrm{R}_{\mathrm{ac}}$ \\
\hline $5 \mathrm{mg}$ BID & $21.4(84)$ & $138(78)$ & $4.04(3.93,7.70)$ & $1.37(28)$ \\
\hline
\end{tabular}

Preliminary antitumor activity

The majority of patients ( $n=16 ; 89 \%$ ) had disease progression before enrollment. Following treatment with axitinib, a reduction in tumor size was observed in 11 of $17(65 \%)$ evaluable patients who had a target lesion. Tumor size decreased by $\geq 30 \%$ in two patients with colorectal cancer and by $\geq 20 \%$ in two patients (one each with NSCLC and thymic cancer). Additionally, stable disease ( $\geq 24$ weeks) was noted in six patients (one each with colorectal cancer, NSCLC, thymic cancer, synovial sarcoma, esophageal malignant melanoma and pancreatic cancer).

\section{Discussion}

Preliminary population pharmacokinetic analyses of cytokine-refractory $\mathrm{mRCC}$ patients indicated that patients who achieved a higher exposure to axitinib (AUC) while receiving $5 \mathrm{mg}$ BID had higher response rate and longer overall survival. Additionally, retrospective analyses of two clinical trials of axitinib in mRCC demonstrated that patients who developed at least one $\mathrm{dBP}$ measurement $\geq 90 \mathrm{~mm} \mathrm{Hg}$ during therapy had a longer overall survival, higher probability of a partial response and greater decreases in tumor size [15]. These findings suggested that increasing the axitinib dose above a starting dose of $5 \mathrm{mg}$ BID in a select group of patients who tolerate the drug without experiencing hypertension may result in improved efficacy. Thus, in axitinib clinical trials, dose titration from a starting dose of $5 \mathrm{mg}$ BID to $7 \mathrm{mg}$ BID and then to a maximum $10 \mathrm{mg}$ BID is recommended if no treatment-related adverse events grade $>2$ are observed and $\mathrm{BP}$ is $\leq 150 / 90 \mathrm{~mm} \mathrm{Hg}$ for 2 consecutive weeks with axitinib $5 \mathrm{mg}$ BID. However, the pharmacokinetic profile following 5-mg, 7-mg and 10-mg single dosing of axitinib has not been evaluated in either Western or Japanese subjects. In Study 2, axitinib pharmacokinetics following 5-mg, 7-mg and 10-mg single dosing was further evaluated.

Single and continuous dosing of axitinib was generally well tolerated in the Japanese patients studied here. The adverse event profile was similar to that seen in other studies of axitinib in Caucasian populations [5-9, 16]. Toxicities associated with axitinib therapy were generally manageable in the current studies. Major grade 3/4 adverse events included fatigue and hypertension. Fatigue (all grades) occurred in $83 \%$ of patients in the two studies, with grade $3 / 4$ fatigue seen in $42 \%$ of patients in Study 1 . Comparable rates of fatigue were reported by Rini et al [6] in a study of

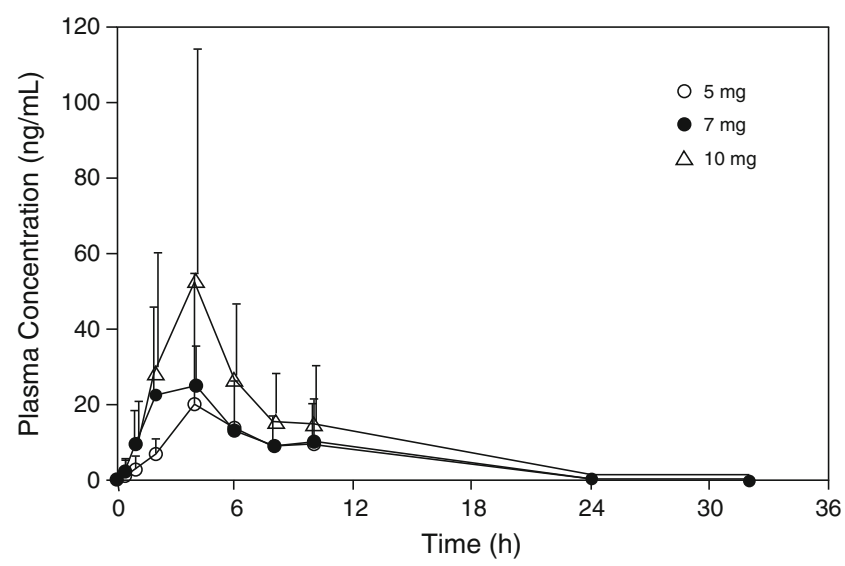

Fig. 2 Axitinib plasma concentration over time following single dosing in Study 2; pharmacokinetic parameters were measured following a single dose of axitinib $(N=6)$ 

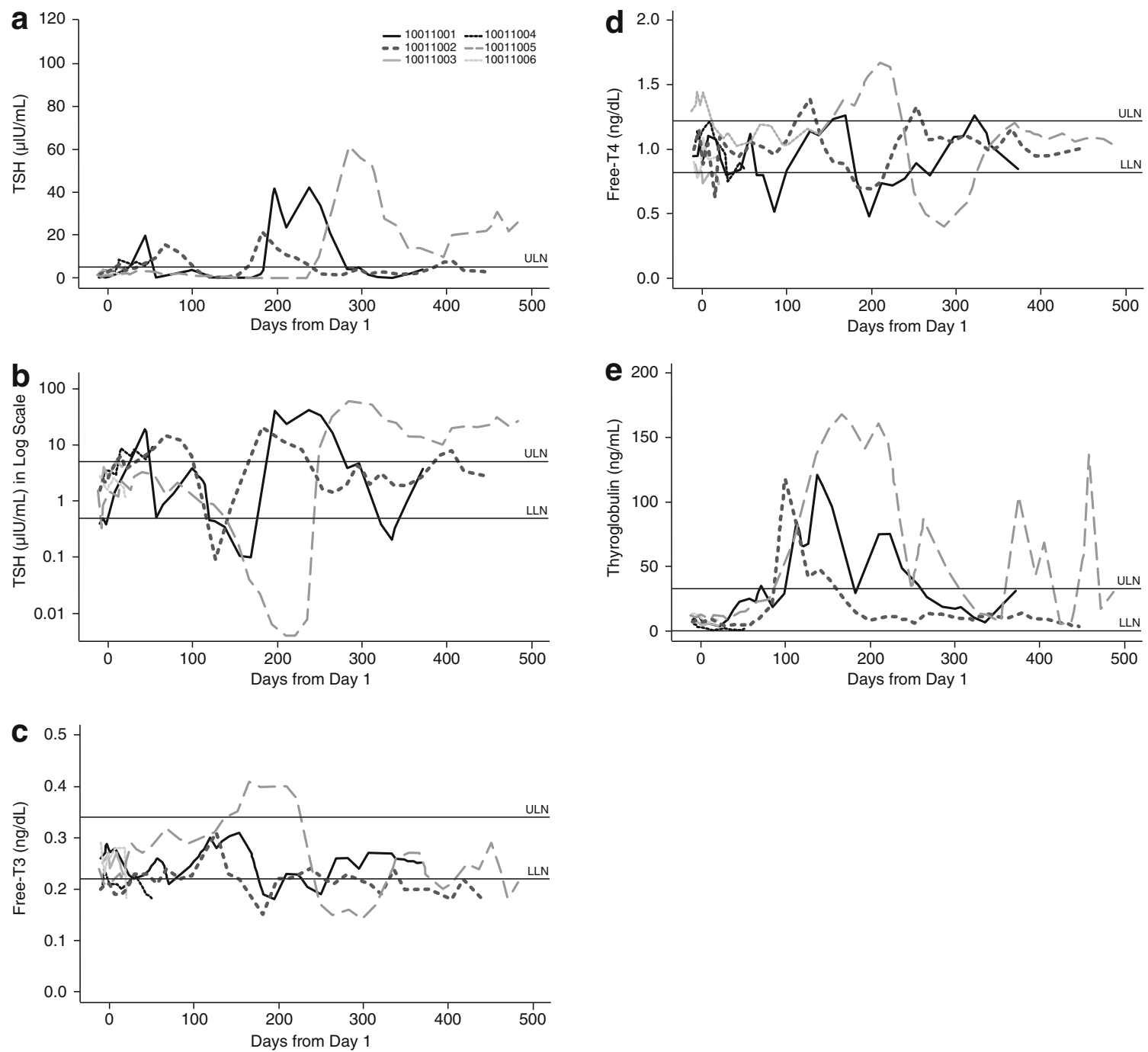

Fig. 3 Changes over time in Study 2 patient laboratory levels: a thyroid-stimulating hormone (absolute values); b thyroid-stimulating hormone (log scale); $\mathbf{c}$ free triiodothyronine (free T3); $\mathbf{d}$ free thyroxine

(free T4); e thyroglobulin. $L L N$ lower limit of normal range; $U L N$ upper limit of normal range

axitinib in sorafenib-refractory RCC. For the six patients in Study 2, proactive thyroid hormone replacement therapy (according to TSH levels) seemed useful to prevent the development of grade $3 / 4$ fatigue related to axitinib treatment. These results suggest that axitinib-related grade 3/4 fatigue may be preventable through careful monitoring of TSH levels and use of thyroid hormone replacement therapy as needed to

Table 5 Treatment-related fatigue in Study 1 and Study 2

\begin{tabular}{lll}
\hline Study & Fatigue & \\
\cline { 2 - 3 } & All grades, $n(\%)$ & Grade 3/4, $n(\%)$ \\
\hline Study $1(N=12)$ & $10(83)$ & $5(42)$ \\
Study 2 $(N=6)^{\text {a }}$ & $5(83)$ & 0 \\
\hline
\end{tabular}

${ }^{\mathrm{a}}$ Four patients received thyroid hormone replacement therapy

maintain a euthyroid state. It is known that anemia is frequently associated with hypothyroidism $[17,18]$. In the present two phase I studies, anemia was not reported as an adverse event. Elevation of hemoglobin was reported in patients treated with VEGFR inhibitors such as axitinib, sunitinib and sorafenib [11, 19-21]. This could obscure the development of anemiaassociated hypothyroidism, and it thus may be difficult to investigate the relationship between anemia and hypothyroidism with the treatment of VEGFR inhibitors.

Elevation of thyroglobulin was observed in all three patients who continued treatment with axitinib for $\geq 3$ months in Study 2 . Thyroglobulin is synthesized by the thyroid gland to produce the thyroid hormones T4 and T3. Increased serum levels of thyroglobulin can suggest Graves' disease or some forms of thyroiditis such as subacute thyroiditis or destructive thyroiditis. The observed changes in thyroglobulin suggest that transient subacute thyroiditis and subsequent hypothyroidism 


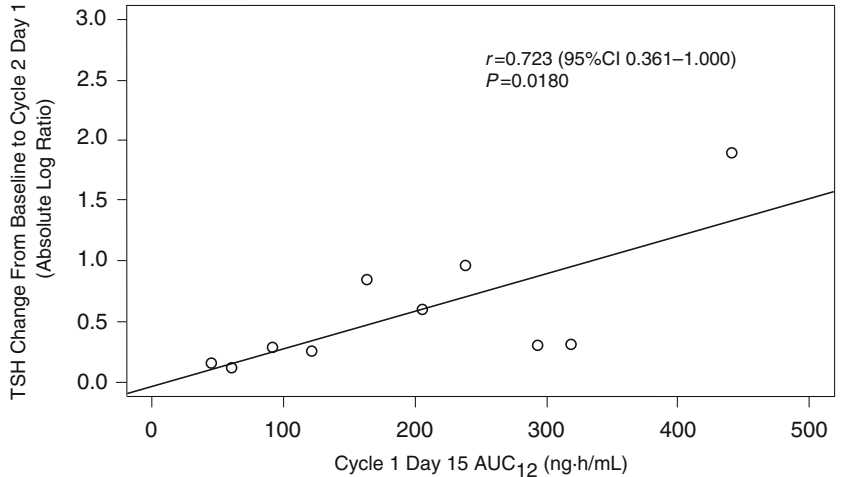

Fig. 4 Relationship between change in thyroid-stimulating hormone (TSH) levels from baseline to cycle 2 day 1 (absolute log ratio) and $\mathrm{AUC}_{12}$ in Studies 1 and 2 (pooled data): $n=10$ - eight patients were excluded (two with abnormal baseline TSH; three received thyroid hormone replacement therapy or had axitinib dose titrated before cycle 2 day 1; two discontinued treatment before cycle 2 day 1 ; and one did not receive cycle 1 day $14 \mathrm{AM}$ dose). $A U C_{12}$ area under the plasma concentration-time curve from time zero to $12 \mathrm{~h}$; $C I$ confidence interval

observed following axitinib treatment could be due to a direct effect of this agent on the thyroid gland.

The mechanism for axitinib-induced hypothyroidism is currently unclear; however, in vitro and in vivo studies have demonstrated that VEGF and VEGFR mRNA and protein are expressed in normal thyroid follicular cells, which is mediated in part by thyroid-stimulating hormone [22-24]. These data suggest it is possible that axitinib, a VEGFR inhibitor, decreases thyroid function by interfering with VEGF function and/or impairing thyroid blood flow, resulting in hypothyroidism. It has been postulated that hypothyroidism associated with sorafenib may be caused by altered thyroid hormone metabolism, increasing type 3 deiodination [25]. Hypothyroidism and thyroid dysfunction are commonly observed with sunitinib (36-85\% of patients) and sorafenib $(\sim 20 \%)[13,26-$ 29]. A study of 16 patients with mRCC treated with sunitinib, sorafenib or axitinib for $\geq 12$ months found that thyroid dysfunction was a common toxicity, occurring in $\sim 40 \%$ of

Table 6 Change in plasma concentration of biomarkers from baseline to cycle 2 day 1 (pooled data from Study 1 and Study 2; $N=16^{\mathrm{a}}$ )

\begin{tabular}{ll}
\hline Biomarker & $\begin{array}{l}\text { \% Change in concentration from baseline } \\
\text { to cycle } 2 \text { day } 1, \text { median (range) }\end{array}$ \\
\hline VEGF & $+242(15$ to 1446$)$ \\
sVEGFR-2 & $-38(-58$ to -10$)$ \\
sVEGFR-3 & $-53(-94$ to -16$)$ \\
sKIT & $-2.9(-24$ to 34$)$
\end{tabular}

${ }^{\mathrm{a}}$ Two patients who discontinued treatment before cycle 2 day 1 were excluded

sKIT soluble stem cell factor receptor; $V E G F$ vascular endothelial growth factor; $s V E G F R-2 / 3$ soluble VEGF receptor-2/3

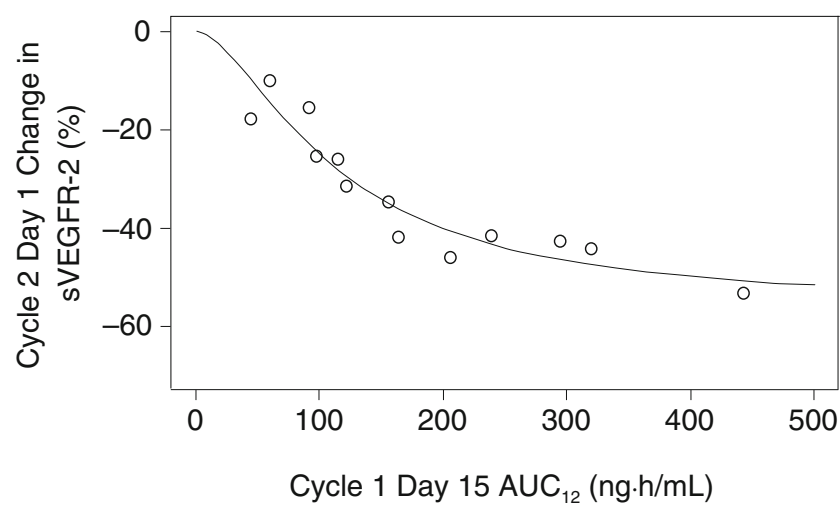

Fig. 5 Relationship between percentage change in soluble VEGFR-2 level from baseline to cycle 2 day 1 and $\mathrm{AUC}_{12}$ in Studies 1 and 2 (pooled data): $n=13$ - five patients were excluded (two discontinued treatment before cycle 2 day 1; two had axitinib dose titrated before cycle 2 day 1 ; and one did not receive cycle 1 day 14 AM dose). $A U C_{12}$ area under the plasma concentration-time curve from time zero to $12 \mathrm{~h}$; VEGFR-2 vascular endothelial growth factor receptor-2

patients [30]. Development of severe hypothyroidism associated with use of these agents in $\mathrm{mRCC}$ is infrequent, however, and typically can be corrected by use of thyroid hormone replacement therapy. Regular monitoring of thyroid-function abnormalities and use of thyroid hormone replacement therapy in patients receiving axitinib and related tyrosine kinase inhibitors is therefore indicated.

In Study 2, it was possible to maintain a euthyroid state in hypothyroid patients with careful monitoring of TSH and thyroid hormone dose adjustments. The decreases in sensitive TSH below the lower limit of normal range during thyroid hormone replacement therapy were not associated with any symptoms of hyperthyroidism and were successfully managed with temporary interruption or dose reduction of thyroid hormone.

The time-course of change in TSH levels and that of total cholesterol appeared to be correlated in some patients. This finding suggests that the observed changes in total cholesterol values were due to axitinib-related hypothyroidism.

A correlation between occurrence of hypothyroidism and progression-free survival was seen in some studies of sunitinib in mRCC [31, 32]. Tyrosine kinase inhibitor-induced thyroid dysfunction, therefore, has been proposed as a possible surrogate marker of efficacy with antiangiogenic tyrosine kinase inhibitors, rather than being viewed as an unwanted toxicity $[31,33]$. In the current phase I studies, increased TSH levels were highly correlated with exposure to axitinib $(r=0.72)$. Additionally, a significant correlation was noted between change in sVEGFR-2 level and exposure to axitinib $(r=-0.94)$. Other clinical trials of axitinib have also observed preferential decreases in sVEGFR levels in patients with thyroid cancer [8] or poorprognosis acute myeloid leukemia/myelodysplastic syndrome [16]. These data suggest that both sVEGFR-2 and TSH levels may serve as biomarkers of axitinib exposure. 
In this report, we used pooled data from two phase I studies to evaluate safety, antitumor activity and biomarkers, and thus could have potential biases for these results. However, eligibility criteria in these two phase I studies were similar, and the interval for patient visit, laboratory test and tumor assessment was the same between the two protocols. The same sample collection point for pharmacokinetics and biomarkers was used to explore the correlation between TSH or sVEGFR-2 level and axitinib exposure. In addition, a standardized case report form was used for data collection in both studies, which may reduce measurement and/or reporting biases. Therefore, biases introduced by using pooled data to evaluate these data could not be substantial.

In conclusion, these results demonstrate that single and continuous dosing of axitinib were generally well tolerated in Japanese cancer patients. While axitinib caused thyroid dysfunction (hypo- and hyperthyroidism), grade $3 / 4$ fatigue and hypothyroidism appear to be controllable by the use of thyroid hormone replacement therapy. Since changes in sVEGFR2 and TSH levels correlated with axitinib plasma levels, both sVEGFR2 and TSH may potentially act as biomarkers of axitinib exposure. Axitinib showed antitumor activity across multiple tumor types in Japanese patients with solid tumors. Further clinical investigation is necessary to clarify the clinical usefulness of these potential biomarkers.

Acknowledgments Editorial assistance was provided by Larry Rosenberg, PhD, at UBC Scientific Solutions, which was funded by Pfizer Inc and supported by Gamal ElSawah, MD, Pfizer Medical Affairs. This study was sponsored by Pfizer Japan Inc.

Yutaka Fujiwara, Naomi Kiyota, Naoko Chayahara and Toru Mukohara have nothing to disclose. Akiyuki Suzuki and Yoshiko Umeyama are employees of Pfizer and own stock in Pfizer. Hironobu Minami received research funds and honoraria from Pfizer Japan Inc.

Open Access This article is distributed under the terms of the Creative Commons Attribution Noncommercial License which permits any noncommercial use, distribution, and reproduction in any medium, provided the original author(s) and source are credited.

\section{References}

1. Kelly RJ, Rixe O (2010) Axitinib (AG-013736). Recent Results Cancer Res 184:33-44

2. Choueiri TK (2008) Axitinib, a novel anti-angiogenic drug with promising activity in various solid tumors. Curr Opin Investig Drugs 9:658-671

3. Hu-Lowe DD, Zou HY, Grazzini ML, Hallin ME, Wickman GR, Amundson K, Chen JH, Rewolinski DA, Yamazaki S, Wu EY, McTigue MA, Murray BW, Kania RS, O'Connor P, Shalinsky DR, Bender SL (2008) Nonclinical antiangiogenesis and antitumor activities of axitinib (AG-013736), an oral, potent, and selective inhibitor of vascular endothelial growth factor receptor tyrosine kinases 1, 2, 3. Clin Cancer Res 14:7272-7283. doi:10.1158/1078-0432.CCR-08-0652
4. Wilmes LJ, Pallavicini MG, Fleming LM, Gibbs J, Wang D, Li KL, Partridge SC, Henry RG, Shalinsky DR, Hu-Lowe D, Park JW, McShane TM, Lu Y, Brasch RC, Hylton NM (2007) AG-013736, a novel inhibitor of VEGF receptor tyrosine kinases, inhibits breast cancer growth and decreases vascular permeability as detected by dynamic contrast-enhanced magnetic resonance imaging. Magn Reson Imaging 25:319-327. doi:10.1016/j.mri.2006.09.041

5. Rixe O, Bukowski RM, Michaelson MD, Wilding G, Hudes GR, Bolte O, Motzer RJ, Bycott P, Liau KF, Freddo J, Trask PC, Kim S, Rini BI (2007) Axitinib treatment in patients with cytokinerefractory metastatic renal-cell cancer: a phase II study. Lancet Oncol 8:975-984. doi:10.1016/S1470-2045(07)70285-1

6. Rini BI, Wilding G, Hudes G, Stadler WM, Kim S, Tarazi J, Rosbrook B, Trask PC, Wood L, Dutcher JP (2009) Phase II study of axitinib in sorafenib-refractory metastatic renal cell carcinoma. J Clin Oncol 27:4462-4468. doi:10.1200/JCO.2008.21.7034

7. Schiller JH, Larson T, Ou SH, Limentani S, Sandler A, Vokes E, Kim S, Liau K, Bycott P, Olszanski AJ, von Pawel J (2009) Efficacy and safety of axitinib in patients with advanced nonsmall-cell lung cancer: results from a phase II study. J Clin Oncol 27:3836-3841. doi:10.1200/JCO.2008.20.8355

8. Cohen EE, Rosen LS, Vokes EE, Kies MS, Forastiere AA, Worden FP, Kane MA, Sherman E, Kim S, Bycott P, Tortorici M, Shalinsky DR, Liau KF, Cohen RB (2008) Axitinib is an active treatment for all histologic subtypes of advanced thyroid cancer: results from a phase II study. J Clin Oncol 26:4708-4713. doi:10.1200/JCO.2007.15.9566

9. Fruehauf J, Lutzky J, McDermott D, Brown CK, Meric J-B, Rosbrook B (2010) Multicenter, phase II study of axitinib (AG-013736), an oral and selective inhibitor of VEGFR 1,2,3, in patients with metastatic melanoma. Clin Cancer Res (in press)

10. Rugo HS, Stopeck A, Joy AA, Chan S, Verma S, Lluch S (2007) A randomized, double-blind phase II study of the oral tyrosine kinase inhibitor (TKI) axitinib (AG-013736) in combination with docetaxel (DOC) compared to DOC plus placebo (PL) in metastatic breast cancer [Abstract 1003]. 43rd Annual Meeting of the American Society of Clinical Oncology (ASCO); Chicago, Illinois, June 1-5, 2007

11. Mukohara T, Nakajima H, Mukai H, Nagai S, Itoh K, Umeyama Y, Hashimoto J, Minami H (2010) Effect of axitinib (AG-013736) on fatigue, thyroid-stimulating hormone, and biomarkers: a phase I study in Japanese patients. Cancer Sci 101:963-968. doi:10.1111/j.1349-7006.2009.01465.x

12. Pithavala YK, Mount J, Toh M, Garrett M, Hee B, Selaru P, Klamerus KJ (2008) Effect of food on the pharmacokinetics of axitinib (AG-013736) in healthy volunteers. Annual Meeting of the American Association of Cancer Research (AACR); San Diego, CA, April 12-16, 2008

13. Rini BI, Tamaskar I, Shaheen P, Salas R, Garcia J, Wood L, Reddy S, Dreicer R, Bukowski RM (2007) Hypothyroidism in patients with metastatic renal cell carcinoma treated with sunitinib. J Natl Cancer Inst 99:81-83. doi:10.1093/jnci/djk008

14. Therasse P, Arbuck SG, Eisenhauer EA, Wanders J, Kaplan RS, Rubinstein L, Verweij J, Van Glabbeke M, van Oosterom AT, Christian MC, Gwyther SG (2000) New guidelines to evaluate the response to treatment in solid tumors. European Organization for Research and Treatment of Cancer, National Cancer Institute of the United States, National Cancer Institute of Canada. J Natl Cancer Inst 92:205-216. doi:10.1093/ jnci/92.3.205

15. Rixe O, Dutcher J, Motzer R, Wilding G, Stadler WM, Garrett M, Pithavala Y, Kim S, Tarazi J, I. RB (2009) Diastolic blood pressure (dBP) and pharmacokinetics (PK) as predictors of axitinib efficacy in metastatic renal cell cancer (mRCC) [Abstract 5045]. 44th Annual Meeting of the American Society of Clinical Oncology (ASCO); Chicago, Illinois, May 30-June 3, 2008 
16. Giles FJ, Bellamy WT, Estrov Z, O'Brien SM, Verstovsek S, Ravandi F, Beran M, Bycott P, Pithavala Y, Steinfeldt H, Reich SD, List AF, Yee KW (2006) The anti-angiogenesis agent, AG013736, has minimal activity in elderly patients with poor prognosis acute myeloid leukemia (AML) or myelodysplastic syndrome (MDS). Leuk Res 30:801-811. doi:10.1016/j.leukres.2005.10.024

17. Dawson MA, Yarbro JW (1970) Anemia in hypothyroidism. South Med J 63:966-970

18. Kosenli A, Erdogan M, Ganidagli S, Kulaksizoglu M, Solmaz S, Kosenli O, Unsal C, Canataroglu A (2009) Anemia frequency and etiology in primary hypothyroidism. Society for Endocrinology BES 2009 Endocrine Abstracts; Harrogate, UK, 16-19 March 2009

19. Alexandre I, Billemont B, Meric JB, Richard S, Rixe O (2009) Axitinib induces paradoxical erythropoietin synthesis in metastatic renal cell carcinoma. J Clin Oncol 27:472-473. doi:10.1200/ JCO.2008.20.1087

20. van der Veldt AA, Boven E, Vroling L, Broxterman HJ, van den Eertwegh AJ, Haanen JG (2009) Sunitinib-induced hemoglobin changes are related to the dosing schedule. J Clin Oncol 27:13391340. doi:10.1200/JCO.2008.20.6151

21. Alexandrescu DT, McClure R, Farzanmehr H, Dasanu CA (2008) Secondary erythrocytosis produced by the tyrosine kinase inhibitors sunitinib and sorafenib. J Clin Oncol 26:4047-4048. doi:10.1200/JCO.2008.18.3525

22. Vesely D, Astl J, Lastuvka P, Matucha P, Sterzl I, Betka J (2004) Serum levels of IGF-I, HGF, TGFbeta1, bFGF and VEGF in thyroid gland tumors. Physiol Res 53:83-89

23. Hoffmann S, Glaser S, Wunderlich A, Lingelbach S, Dietrich C, Burchert A, Muller H, Rothmund M, Zielke A (2006) Targeting the EGF/VEGF-R system by tyrosine-kinase inhibitors-a novel antiproliferative/antiangiogenic strategy in thyroid cancer. Langenbecks Arch Surg 391:589-596. doi:10.1007/s00423-0060104-y

24. Keefe SM, Cohen MA, Brose MS (2010) Targeting vascular endothelial growth factor receptor in thyroid cancer: the intracellular and extracellular implications. Clin Cancer Res 16:778-783. doi:10.1158/1078-0432.CCR-08-2743

25. Abdulrahman RM, Verloop H, Hoftijzer H, Verburg E, Hovens GC, Corssmit EP, Reiners C, Gelderblom H, Pereira AM, Kapiteijn E, Romijn JA, Visser TJ, Smit JW (2010) Sorafenibinduced hypothyroidism is associated with increased type 3 deiodination. J Clin Endocrinol Metab 95:3758-3762. doi:10.1210/jc.2009-2507

26. Tamaskar I, Bukowski R, Elson P, Ioachimescu AG, Wood L, Dreicer R, Mekhail T, Garcia J, Rini BI (2008) Thyroid function test abnormalities in patients with metastatic renal cell carcinoma treated with sorafenib. Ann Oncol 19:265-268. doi:10.1093/ annonc/mdm 483

27. Miyake H, Kurahashi T, Yamanaka K, Kondo Y, Muramaki M, Takenaka A, Inoue TA, Fujisawa M (2009) Abnormalities of thyroid function in Japanese patients with metastatic renal cell carcinoma treated with sorafenib: a prospective evaluation. Urol Oncol 28:515-519. doi:10.1016/j.urolonc.2009.08.011

28. Shepard DR, Garcia JA (2009) Toxicity associated with the longterm use of targeted therapies in patients with advanced renal cell carcinoma. Expert Rev Anticancer Ther 9:795-805. doi:10.1586/ era.09.29

29. Torino F, Corsello SM, Longo R, Barnabei A, Gasparini G (2009) Hypothyroidism related to tyrosine kinase inhibitors: an emerging toxic effect of targeted therapy. Nat Rev Clin Oncol 6:219-228. doi:10.1038/nrclinonc.2009.4

30. Vakkalanka BK, Elson P, Wood L, Dreicer R, Garcia JA, Bukowski RM, Rini BI (2008) Long term toxicity of tyrosine kinase inhibitors (TKIs) in patients with metastatic clear cell renal cell carcinoma (RCC). 44th Annual Meeting of the American Society of Clinical Oncology (ASCO); Chicago, Illinois, May 30June 3, 2008

31. Wolter P, Stefan C, Decallonne B, Dumez H, Fieuws S, Wildiers H, Clement P, Debaere D, Van Oosterom A, P. S (2008) Evaluation of thyroid dysfunction as a candidate surrogate marker for efficacy of sunitinib in patients (pts) with advanced renal cell cancer (RCC). 44th Annual Meeting of the American Society of Clinical Oncology (ASCO); Chicago, Illinois, May 30-June 3, 2008

32. Sabatier R, Gravis G, Deville J, Salem N, Brunelle S, Walz J, Marcy M, Narbonne H, Viens P, Bladou F (2009) Hypothyroidism and survival during sunitinib therapy in metastatic renal cell cancer: A prospective observational analysis. Proceedings of the 2009 Genitourinary Cancers Symposium, Orlando, Florida

33. Schmidinger M, Vogl UM, Bojic M, Lamm W, Heinzl H, Haitel A, Clodi M, Kramer G, Zielinski CC (2010) Hypothyroidism in patients with renal cell carcinoma: blessing or curse?. Cancer Sep 15 [Epub ahead of print] doi:10.1002/cncr.25422 